\title{
Survival and prognosis factors in systemic sclerosis: data of a French multicenter cohort, systematic review, and meta- analysis of the literature
}

M. R. Pokeerbux ${ }^{1,2,3,4}$, J. Giovannelli ${ }^{1,2,3,4}$, L. Dauchet ${ }^{5 \dagger}$, L. Mouthon ${ }^{6 \dagger}$, C. Agard ${ }^{7}$, J. C. Lega ${ }^{8,9}$, Y. Allanore ${ }^{10}$, P. Jego ${ }^{11}$, B. Bienvenu ${ }^{12}$, S. Berthier ${ }^{13}$, A. Mekinian ${ }^{14}$, E. Hachulla $a^{1,2,3,4}$ and D. Launay ${ }^{1,2,3,4^{*}}$

\begin{abstract}
Background: Data on survival and prognosis factors in incident cohorts are scarce in systemic sclerosis (SStc). To describe survival, standardized mortality ratio (SMR), and prognosis factors in systemic sclerosis (SSC), we analyzed a multicenter French cohort of incident patients and performed a systematic review of the literature and meta-analysis.

Methods: A multicenter, French cohort study was conducted between January 1, 2000, and December 31, 2013. Patients were followed-up until July 1, 2016.

A systematic review of the literature was carried out in MEDLINE and EMBASE up to July 2017. Meta-analysis was performed using all available data on SMR and hazard ratios of prognosis factors.

Results: A total of 625 patients (493 females, 446 lcSSc) were included. During the study period, 104 deaths (16.6\%) were recorded and 133 patients were lost to follow-up. Overall survival rates at 1,3,5, and 10 years from diagnosis were 98.0\%, 92.5\%, 85.9\%, and 71.7\% respectively in the French cohort. Overall SMR was 5.73 (95\% Cl 4.68-6.94). Age at diagnosis > 60 years, diffuse cutaneous SSc, scleroderma renal crisis, dyspnea, 6-min walking distance (6MWD), forced vital capacity $<70 \%$, diffusing capacity of the lungs for carbon monoxide $<70 \%$, pulmonary hypertension (PH), telangiectasia, valvular disease, malignancy, anemia, and CRP $>8 \mathrm{mg} / \mathrm{l}$ were associated with a poorer survival after adjustment.

Eighteen studies (11,719 patients) were included in the SMR meta-analysis and 36 studies (26,187 patients) in the prognosis factor analysis. Pooled SMR was 3.45 (95\%Cl 3.03-3.94). Age at disease onset, male sex, African origin, diffuse cutaneous SSc, anti-Scl70 antibodies, cardiac and renal involvement, interstitial lung disease, PH, and malignancy were significantly associated with a worse prognosis. Anti-centromere antibodies were associated with a better survival.

Conclusions: Overall, our study highlights a high mortality rate in SSc patients and confirms previously described prognosis factors related to skin extension and organ involvement while identifying additional prognosis factors such as autoantibody status, telangiectasia, 6MWD, and valvular disease.
\end{abstract}

Keywords: Systemic sclerosis, Prognosis factors, Survival, Meta-analysis

\footnotetext{
*Correspondence: launayd@gmail.com; david.launay@univ-lille.fr

${ }^{+} \mathrm{L}$ Dauchet and $\mathrm{L}$ Mouthon contributed equally to this work.

${ }^{1}$ University of Lille, U995 - LIRIC - Lille Inflammation Research International

Center, F-59000 Lille, France

${ }^{2}$ INSERM, U995, F-59000 Lille, France

Full list of author information is available at the end of the article
}

(c) The Author(s). 2019 Open Access This article is distributed under the terms of the Creative Commons Attribution 4.0 International License (http://creativecommons.org/licenses/by/4.0/), which permits unrestricted use, distribution, and reproduction in any medium, provided you give appropriate credit to the original author(s) and the source, provide a link to the Creative Commons license, and indicate if changes were made. The Creative Commons Public Domain Dedication waiver (http://creativecommons.org/publicdomain/zero/1.0/) applies to the data made available in this article, unless otherwise stated. 


\section{Background}

Systemic sclerosis (SSc) is an autoimmune disease, characterized by microvascular damage, dysregulation of both innate and adaptative immunity, and fibrosis of multiple organs. The causes of SSc-related deaths evolved over the last decades, with cardiac and respiratory complications currently being the leading causes of death $[1,2]$.

Prior cohort studies comparing contemporary and historical cohort have suggested an improvement of survival rates over time $[1,3,4]$. Yet, two recent meta-analyses have reported that standardized mortality ratio (SMR) was stable over time $[5,6]$. Moreover, most of the observational studies investigating mortality in SSc included prevalent cases, which may result in an underestimation of mortality due to a survivor bias. Data on survival in incident cohorts are scarce in SSc [7-12].

Previous studies [7, 13-26] reported risk factors for poor survival in SSc such as male sex, diffuse cutaneous subtype, and specific organ involvement. Recently, Elhai et al. developed a prognostic score from the large EUSTAR database, which accurately predicts 3-year mortality [2]. To our knowledge, two meta-analyses combined the results of the available literature to assess prognosis factors [5, 27]. However, these meta-analyses did not assess prognosis factors such as auto-antibody profile, genetic background, and cancer and did not assess the influence of prevalent versus incident cases.

The aim of the present study was to fill these gaps by assessing survival and prognosis factors in a multicenter French cohort of incident SSc patients and by performing a systematic review of the literature and meta-analysis including all available prognosis factors and SMR.

\section{Methods}

\section{Population}

The French National Scleroderma Cohort includes 42 centers. The present analysis was restricted to five university hospitals, Lille, Paris (two centers), Nantes, and Lyon to ensure better quality of data, especially on survival data. These five centers participated in recruiting about two thirds of the National Cohort. Data were retrospectively collected before 2010 and then prospectively collected.

Patients were included between January 1, 2000, and December 31, 2013, if they met the following inclusion criteria: (i) be aged over 18, (ii) fulfill the ACR 1980 preliminary classification criteria [28] or ACR/EULAR 2013 classification criteria [29] for SSc, (iii) have at least one additional visit after the inclusion visit, and (iv) be incident cases, defined as patients having disease duration from time of diagnosis to enrolment in the study of less than 3 years. Patients were followed-up until July 1, 2016. Patients were considered as lost to follow-up if the vital status could not be ascertained. When possible, the vital status was ascertained by querying death registers at birth town councils.

\section{Collected data and variable definition}

Data collected at the inclusion visit were patient demographics, history of Raynaud phenomenon (RP) and first non-RP symptom, SSc subtype and modified Rodnan skin score (mRSS), auto-antibody profile, and organ involvement.

Disease onset was defined as the time of onset of first non-RP symptom. Interstitial lung disease was diagnosed on HRCT or chest $\mathrm{x}$-ray. Pulmonary function tests including forced vital capacity (FVC) and diffusing capacity of the lungs for carbon monoxide (DLCO) were collected. Six-minute walking distance (6MWD) was collected. Pulmonary hypertension (PH) was suspected on a Doppler echocardiogram when systolic pulmonary arterial pressure (PAP) was estimated to be $>35 \mathrm{mmHg}$ or maximum tricuspid regurgitant jet velocity $>2.8 \mathrm{~m} / \mathrm{s}$. Pulmonary arterial hypertension $(\mathrm{PAH})$ was confirmed by right heart catheterization (RHC) when mean PAP was found to be $\geq$ $25 \mathrm{mmHg}$ at rest, with mean pulmonary arterial wedge pressure $\leq 15 \mathrm{mmHg}$. EKG alterations, left ventricular ejection fraction (LVEF), diastolic dysfunction [30], valvular disease (excluding tricuspid valve regurgitation to avoid confounding with $\mathrm{PH}$ ), and pericarditis were recorded according to the American Society of Echocardiography and European Association of Cardiovascular Imaging guidelines [30]. Scleroderma renal crisis was defined as new onset hypertension $>150 / 85 \mathrm{mmHg}$ associated with a decrease in renal function or manifestations of malignant hypertension. Gastrointestinal tract involvement included reflux, dysmotility, constipation, or diarrhea; signs of bacterial overgrowth and/or malabsorption; and abnormal manometry and/or endoscopy test. Muscle involvement included myalgia and/ or muscle weakness and/or elevation of creatinine kinase (CPK). Joint involvement included arthralgia, synovitis, and/or tendon friction rubs. Anemia was defined as a hemoglobin level $<12 \mathrm{~g} / \mathrm{dl}$. Smoking included self-reported current or former cigarette smoking.

\section{Systematic review and meta-analysis}

The meta-analysis was conducted according to MOOSE guidelines [31]. MEDLINE and EMBASE databases were queried by two of the authors (MRP and DL) using the following search terms: ((systemic sclerosis [Title]) OR (scleroderma, systemic[Title])) AND ((death) OR (mortality) OR (prognosis) OR (survival)). Cochrane did not retrieve additional abstracts. All records published before July 1, 2017, were included in the search. Language was restricted to English or French. Reference list of selected studies was hand-searched for additional relevant studies to be included in the meta-analysis. 
Two of the authors (MRP and DL) independently screened the titles and abstracts of the retrieved records to identify eligible articles. The full text of eligible articles was read for inclusion in the meta-analysis. Selected articles were compared, and in case of disagreement, decisions were made by consensus.

Cohort studies of unselected adult SSc patients assessing routine clinical and laboratory prognosis factors and SMR were included. Studies which included patients diagnosed with SSc overlap with other connective tissue diseases were excluded.

Studies from the same centers were included if their respective study periods were different. If, for the same center, two studies covered an overlapping study period, data from the largest cohort were kept in the analysis. A study was recorded as an incident according to the authors' definition.

Quality of the studies was assessed using the NewcastleOttawa scale [32].

Data were extracted and entered into a predefined spreadsheet table which included the following items: study design, length of follow-up, definition of disease onset, disease duration, SMR, and adjusted or, if unavailable, unadjusted hazard ratios (HR) for each studied prognosis factor.

\section{Data analysis}

Characteristics of the population were described using mean \pm standard deviation or median (interquartile range $(\mathrm{IQR}))$ in case of non-normality, for quantitative variables, and number (percentage) for qualitative variables. Comparisons between limited cutaneous SSc (lcSSc) and diffuse cutaneous SSc (dcSSc) patients were conducted using the Student $t$ test or Wilcoxon test in case of non-normality for quantitative variables and Fisher's exact test for qualitative variables.

Survival was estimated from diagnosis using the Kaplan-Meier method. Prognosis factors were assessed by Cox regression analysis in the non-adjusted analysis and subsequently adjusted for age, sex, and SSc subtype. The assumption that hazard ratios were constant over time was verified. SMR was calculated as the ratio of observed death in the cohort to the number of death of the French age/sex-matched population in 2014.

We calculated weighted pooled summary estimates of SMR and HR of prognosis factors. For each meta-analysis, we used the DerSimonian and Laird method. Accordingly, studies were considered to be a random sample from a population of studies. Heterogeneity was assessed using an $I^{2}$ statistic and a chi-square heterogeneity statistic. A random-effects model was used to combine data. The overall effect was estimated using a weighted average of individual effects, with weights inversely proportional to variance in observed effects. Publication bias was evaluated with a funnel plot and Egger's test. The pooled SMR and HR were estimated with 95\% confidence interval (CI). Meta-regression was used to assess the impact of mid-cohort year, the proportion of males, the proportion of diffuse cutaneous forms, and the prevalence of anti-Scl70 antibodies on SMR. The impact of diagnosis of $\mathrm{PH}$ by $\mathrm{RHC}$ on the association of $\mathrm{PH}$ with mortality was evaluated. Separate analyses were performed for (i) SMR according to whether a given study included incident cases only and (ii) HR of $\mathrm{PH}$ diagnosed by either echocardiography and/or RHC and PH diagnosed by RHC.

All analyses were performed using $\mathrm{R}$ software with the survival and metafor packages. $p$ values less than 0.05 were considered significant.

\section{Results}

French cohort study

Baseline characteristics

A total of 625 patients (493 females, 446 lcSSc) were included. Mean age at disease onset was $52.7 \pm 14.9$ years. The median disease duration from disease onset was 0.8 (IQR 2.2) years. Median follow-up time was 4.4 (IQR 5.3) years. The baseline characteristics are shown in Table 1.

\section{Survival and standardized mortality ratio}

During the study period, 104 deaths (16.6\%) were recorded and 133 patients were lost to follow-up. Overall survival rates at $1,3,5,10$, and 15 years from diagnosis were $98.0 \%$ (95\% CI 96.9-99.1\%), 92.5\% (90.4-94.7\%), $85.9 \%$ (82.8-89.1\%), 71.7\% (66.3-77.5\%), and 53\% (33.8$83.4 \%)$ respectively. Survival rates for the diffuse and limited cutaneous subtypes are shown in Fig. 1 and in Additional file 1: Table S1. Overall SMR was 5.73 (95\% CI 4.68-6.94).

\section{Prognosis factors}

Age of diagnosis $>60$ years, dcSSc subtype, telangiectasia, scleroderma renal crisis, severe dyspnea NYHA functional classes III and IV, a shorter distance at the $6 \mathrm{MWD}, \mathrm{FVC}<70 \%$, DLCO $<70 \%, \mathrm{PH}$, valvular disease, anemia, CRP $>8 \mathrm{mg} / \mathrm{l}$, and cancer were associated with a worse prognosis (Table 2).

No association was found for digital ulcers, gastrointestinal, articular, muscular involvement, and specific auto-antibodies after adjustment.

Male sex showed a trend towards worse outcome, but without reaching statistical significance $(H R=1.53 ; 95 \%$ CI $0.98-2.39 ; p=0.06$ ).

\section{Meta-analysis: study selection}

A total of 4128 citations were assessed for inclusion. After screening, 244 abstracts were deemed potentially 
Table 1 Demographics and clinical characteristics of 625 patients with SSC at baseline

\begin{tabular}{|c|c|c|c|c|c|}
\hline & $N(N$ for dcSSc) & Total & dcSSc & ICSSC & $p$ \\
\hline \multicolumn{6}{|l|}{ Demographics } \\
\hline Female sex & $625 / 179$ & $493(79)$ & $124(69)$ & $369(83)$ & $<0.001$ \\
\hline Age at first RP (years) & $554 / 155$ & $45.4 \pm 15.7$ & $45.8 \pm 15.7$ & $45.3 \pm 15.8$ & 0.736 \\
\hline Age at first non-RP symptom (years) & $502 / 160$ & $50.6 \pm 14.5$ & $48.5 \pm 14.4$ & $51.5 \pm 14.4$ & 0.031 \\
\hline Age at diagnosis (years) & $625 / 179$ & $52.7 \pm 14.9$ & $49.5 \pm 14.5$ & $53.9 \pm 14.9$ & $<0.001$ \\
\hline Disease duration from first non-RP symptom to diagnosis (years) & $499 / 160$ & $0.8[2.2]$ & $0.7[1.4]$ & $0.9[2.7]$ & 0.042 \\
\hline Follow-up time from inclusion to death or last visit (years) & $625 / 179$ & $4.4[5.3]$ & $4.0[5.2]$ & $4.8[5.3]$ & 0.023 \\
\hline \multicolumn{6}{|l|}{ Genetic background } \\
\hline European & $503 / 147$ & $453(90)$ & $118(80)$ & $335(94)$ & $<0.001$ \\
\hline African & $503 / 147$ & $50(10)$ & $29(20)$ & $21(6)$ & $<0.001$ \\
\hline \multicolumn{6}{|l|}{ Skin involvement } \\
\hline ICSSC & $625 / 179$ & $446(71)$ & - & - & \\
\hline mRSS & $342 / 123$ & $9.2 \pm 10.2$ & $19.6 \pm 10.1$ & $3.5 \pm 3.6$ & $<0.001$ \\
\hline Telangiectasia & $572 / 160$ & $264(46)$ & $65(41)$ & $199(48)$ & 0.112 \\
\hline Calcinosis & $549 / 152$ & $64(12)$ & $7(5)$ & $57(14)$ & $<0.001$ \\
\hline Digital ulcers (past or active) & $538 / 145$ & $161(30)$ & $66(46)$ & $95(24)$ & $<0.001$ \\
\hline \multicolumn{6}{|l|}{ Pulmonary involvement } \\
\hline NYHA & & & & & 0.702 \\
\hline Classes I-II & $515 / 150$ & $425(83)$ & $122(81)$ & $303(83)$ & \\
\hline Classes III-IV & $515 / 150$ & $90(17)$ & $28(19)$ & $62(17)$ & \\
\hline 6MWD (meters) & $274 / 61$ & $427 \pm 127$ & $432 \pm 135$ & $425 \pm 125$ & 0.705 \\
\hline TLC $<70 \%$ predicted & $472 / 145$ & $64(14)$ & $33(23)$ & $31(9)$ & $<0.001$ \\
\hline FVC $<70 \%$ predicted & $475 / 148$ & $82(17)$ & $44(30)$ & $38(12)$ & $<0.001$ \\
\hline DLCO $<70 \%$ predicted & $471 / 141$ & $249(53)$ & $102(72)$ & $147(45)$ & $<0.001$ \\
\hline Interstitial lung disease & $582 / 166$ & $262(45)$ & $115(69)$ & $147(35)$ & $<0.001$ \\
\hline PH (echo. and/or RHC) & $547 / 157$ & $67(12)$ & $18(11)$ & $49(13)$ & 0.775 \\
\hline sPAP (echo.) & & & & & 0.004 \\
\hline$<35 \mathrm{mmHg}$ & $397 / 118$ & $307(77)$ & $89(75)$ & $218(78)$ & \\
\hline $35-46 \mathrm{mmHg}$ & $397 / 118$ & $43(11)$ & $21(18)$ & $22(8)$ & \\
\hline$>46 \mathrm{mmHg}$ & $397 / 118$ & $47(12)$ & $8(7)$ & $39(14)$ & \\
\hline $\mathrm{PAH}(\mathrm{RHC})$ & $490 / 116$ & $40(8)$ & $4(3)$ & $36(10)$ & 0.033 \\
\hline \multicolumn{6}{|l|}{ Heart involvement } \\
\hline Arrhythmia & $519 / 150$ & $17(3)$ & $5(3)$ & $12(3)$ & 1.000 \\
\hline AV block & $512 / 146$ & $7(1)$ & $4(3)$ & $3(1)$ & 0.106 \\
\hline BB block & $479 / 128$ & $16(3)$ & $6(5)$ & $10(3)$ & 0.388 \\
\hline LVEF (\%) & $402 / 102$ & $64.9 \pm 7.1$ & $65.5 \pm 8.6$ & $64.7 \pm 6.6$ & 0.251 \\
\hline Diastolic dysfunction & $423 / 110$ & $20(5)$ & $6(5)$ & $14(4)$ & 0.613 \\
\hline Pericarditis & $478 / 136$ & $32(7)$ & $14(10)$ & $18(5)$ & 0.066 \\
\hline Valvular disease & $430 / 111$ & $29(7)$ & $5(5)$ & $24(8)$ & 0.380 \\
\hline \multicolumn{6}{|l|}{ Renal involvement } \\
\hline $\mathrm{GFR}<80 \mathrm{ml} / \mathrm{min}$ & $459 / 136$ & $179(39)$ & $38(28)$ & $141(44)$ & 0.002 \\
\hline Scleroderma renal crisis & $428 / 139$ & $44(10)$ & $31(22)$ & $13(5)$ & $<0.001$ \\
\hline Gastrointestinal involvement & $611 / 172$ & $429(70)$ & $135(78)$ & $294(67)$ & 0.006 \\
\hline $\mathrm{BMI}\left(\mathrm{kg} / \mathrm{m}^{2}\right)$ & $514 / 159$ & $24.4 \pm 5.0$ & $23.6 \pm 4.0$ & $24.7 \pm 5.3$ & 0.016 \\
\hline Albuminemia $<35 \mathrm{~g} / \mathrm{l}$ & $331 / 108$ & $52(16)$ & $28(26)$ & $24(11)$ & $<0.001$ \\
\hline
\end{tabular}


Table 1 Demographics and clinical characteristics of 625 patients with SSc at baseline (Continued)

\begin{tabular}{|c|c|c|c|c|c|}
\hline & $N(N$ for dcSSc) & Total & dcSSc & ICSSC & $p$ \\
\hline Muscular involvement & $604 / 172$ & $137(23)$ & $71(41)$ & $66(15)$ & $<0.001$ \\
\hline CPK > $200 \mathrm{IU} / \mathrm{I}$ & $250 / 82$ & $66(26)$ & $33(40)$ & $33(20)$ & $<0.001$ \\
\hline Joint involvement & $598 / 172$ & $291(49)$ & $127(74)$ & $164(39)$ & $<0.001$ \\
\hline Cancer & $625 / 179$ & $49(8)$ & $17(10)$ & $32(7)$ & 0.327 \\
\hline Hemoglobin & $559 / 163$ & $13.0 \pm 1.6$ & $12.5 \pm 1.6$ & $13.1 \pm 1.5$ & $<0.001$ \\
\hline Anemia & $559 / 163$ & $127(23)$ & $52(32)$ & $75(19)$ & 0.001 \\
\hline $\mathrm{CRP}>8 \mathrm{mg} / \mathrm{l}$ & $470 / 136$ & $118(25)$ & $57(42)$ & $61(18)$ & $<0.001$ \\
\hline \multicolumn{6}{|l|}{ Serologic features } \\
\hline $\mathrm{ACA}$ & $557 / 151$ & $221(40)$ & $6(4)$ & $215(53)$ & $<0.001$ \\
\hline Anti-Scl70 antibodies & $504 / 149$ & $177(35)$ & $90(60)$ & $87(25)$ & $<0.001$ \\
\hline Anti-U1RNP antibodies & $342 / 63$ & $15(4)$ & $4(6)$ & $11(4)$ & 0.492 \\
\hline Anti-RNAP3 antibodies & $345 / 72$ & $18(5)$ & $13(18)$ & $5(2)$ & $<0.001$ \\
\hline Anti-PMScl antibodies & $343 / 62$ & $16(5)$ & $3(5)$ & $13(5)$ & 1.000 \\
\hline Anti-SSa antibodies & $387 / 79$ & $60(16)$ & $20(25)$ & $40(13)$ & 0.014 \\
\hline Anti-SSb antibodies & $338 / 62$ & $11(3)$ & $3(5)$ & $8(3)$ & 0.431 \\
\hline APL antibodies & $441 / 129$ & $31(7)$ & $16(12)$ & $15(5)$ & 0.007 \\
\hline Low complement & $482 / 130$ & $18(4)$ & $6(5)$ & $12(3)$ & 0.589 \\
\hline Smoking & $572 / 158$ & $215(38)$ & $69(44)$ & $146(35)$ & 0.067 \\
\hline
\end{tabular}

Results are expressed as $n(\%)$ for qualitative variables and mean \pm SD or median [IQR] for quantitative variables

$N$ number of patients with available data, ICSSc limited cutaneous systemic sclerosis, mRSS modified Rodnan score, GFR glomerular filtration rate, $A V$ block atrioventricular block, $B B$ block bundle branch block, LVEF left ventricular ejection fraction, $P H$ pulmonary hypertension, $P A H$ pulmonary arterial hypertension, echo echocardiography, $R H C$ right heart catheterization, $6 M W D$ 6-min walking distance, $s P A P$ systolic pulmonary arterial pressure, $T L C$ total lung capacity, FVC forced vital capacity, DLCO diffusing capacity of the lungs for carbon monoxide, CRP C reactive protein, $B M I$ body mass index, $A C A$ anti-centromere antibodies, $A P L$ antiphospholipid antibodies

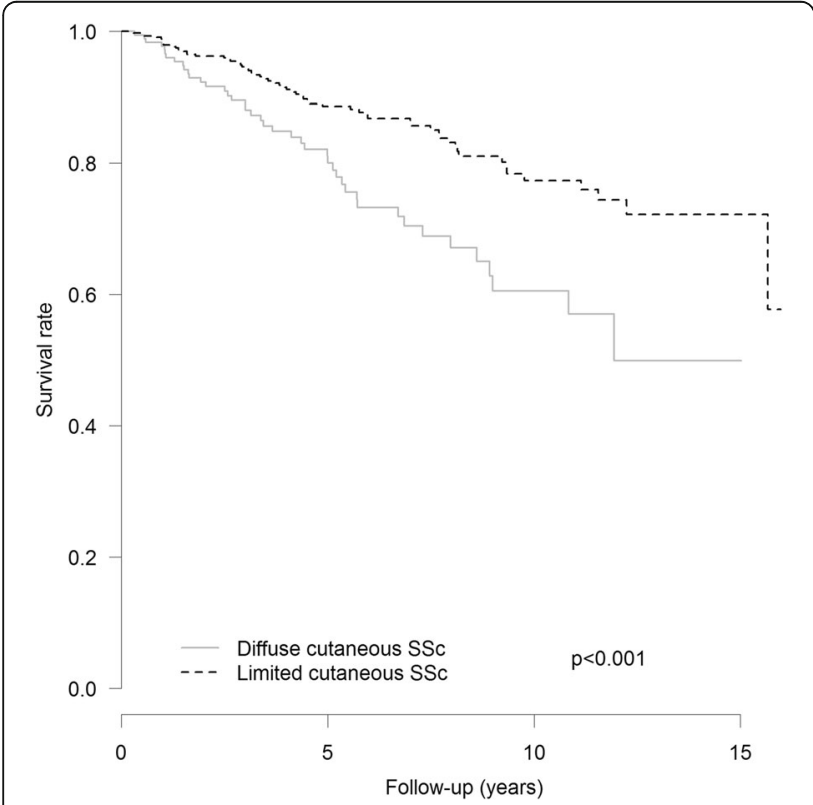

Fig. 1 Kaplan-Meier survival curves from diagnosis for IcSSc and dcSSc patients in the French cohort relevant and the full-text copies were obtained. Of these articles, 44 studies, including our cohort, were included in the meta-analysis (Fig. 2). Eighteen articles were included in the SMR analysis, representing a total population of 11,719 patients [7-12, 15, 22-24, 26, 33-38]. Thirty-six studies were included in the prognosis factor analysis, representing a total of 26,187 patients $[3,7,10-$ $18,20,22-26,34,39-55]$. No study was excluded based on poor quality. The main characteristics of the studies are summarized in Additional file 1: Table S2 and Table S3.

\section{SMR meta-analysis}

The pooled SMR for all studies was 3.45 (95\% CI 3.033.94; $I^{2}=88.8 \% ; p$ (het) $\left.<0.001\right)$. The pooled SMR for studies including only incident patients was 3.64 (95\% CI 3.06-4.34; $I^{2}=82.0 \% ; p$ (het $<0.001$ ), and the pooled SMR for studies including prevalent patients was 3.28 (95\% CI 2.69-3.99; $I^{2}=91.6 \%$; $p$ (het) $<0.001$ ). There was no funnel plot asymmetry, and Egger's test failed to provide any evidence for small study effect, making publication bias unlikely. Meta-regression stratified by study type (incident or prevalent) did not show any association with $\operatorname{SMR}(p=0.461)$, meaning no statistical difference between pooled SMR of incident and prevalent studies. Subsequent analyses were therefore conducted on all 
Table 2 Prognosis factors: non-adjusted and adjusted analysis on age at diagnosis, sex, and SSc subtype in the French cohort

\begin{tabular}{|c|c|c|c|c|}
\hline & Non-adjusted HR & $p$ & Adjusted HR & $p$ \\
\hline \multicolumn{5}{|l|}{ Demographics } \\
\hline Male sex & $2.00(1.31-3.05)$ & 0.001 & $1.53(0.98-2.39)$ & 0.060 \\
\hline Age at diagnosis (per 1 year) & $1.05(1.04-1.07)$ & $<0.001$ & $1.08(1.04-1.12)$ & $<0.001$ \\
\hline Age at diagnostic $>60$ years & $4.97(2.53-9.78)$ & $<0.001$ & $5.79(2.92-11.49)$ & $<0.001$ \\
\hline Disease duration at time of diagnosis (per 1 year) & $1.02(0.97-1.07)$ & 0.542 & $1.01(0.96-1.06)$ & 0.763 \\
\hline African origin (vs. European) & $0.79(0.38-1.62)$ & 0.516 & $0.93(0.43-2.03)$ & 0.864 \\
\hline \multicolumn{5}{|l|}{ Skin involvement } \\
\hline dcSSc subtype (vs. IcSSc) & $2.06(1.39-3.05)$ & $<0.001$ & $2.40(1.58-3.64)$ & $<0.001$ \\
\hline$m R S S>5$ & $1.24(1.12-1.38)$ & $<0.001$ & $1.21(1.03-1.43)$ & 0.022 \\
\hline Past and/or active digital ulcers & $1.22(0.79-1.90)$ & 0.371 & $1.29(0.81-2.04)$ & 0.277 \\
\hline Telangiectasia & $1.64(1.08-2.48)$ & 0.019 & $1.55(1.02-2.35)$ & 0.039 \\
\hline Calcinosis & $1.37(0.79-2.36)$ & 0.260 & $1.22(0.69-2.16)$ & 0.503 \\
\hline \multicolumn{5}{|l|}{ Lung involvement } \\
\hline NYHA class I & - & & - & \\
\hline NYHA class II & $2.68(1.46-4.92)$ & 0.001 & $2.37(1.29-4.36)$ & 0.006 \\
\hline NYHA class III & $17.53(3.97-14.27)$ & $<0.001$ & $6.74(3.53-12.88)$ & $<0.001$ \\
\hline NYHA class IV & $25.76(10.55-62.92)$ & $<0.001$ & $16.61(6.68-41.26)$ & $<0.001$ \\
\hline NYHA classes III-IV (vs. class I) & $4.68(3.07-7.13)$ & $<0.001$ & $4.33(2.82-6.66)$ & $<0.001$ \\
\hline 6MWD (per 100 m) & $0.46(0.36-0.58)$ & $<0.001$ & $0.51(0.39-0.67)$ & $<0.001$ \\
\hline $\mathrm{TLC}<70 \%$ predicted & $3.87(2.36-6.35)$ & $<0.001$ & $3.38(1.96-5.82)$ & $<0.001$ \\
\hline FVC $<70 \%$ predicted & $3.11(1.92-5.02)$ & $<0.001$ & $2.79(1.62-4.80)$ & $<0.001$ \\
\hline DLCO $<70 \%$ predicted & $4.01(2.33-6.89)$ & $<0.001$ & $3.31(1.87-5.88)$ & $<0.001$ \\
\hline Interstitial lung disease & $1.99(1.32-2.99)$ & $<0.001$ & $1.50(0.96-2.34)$ & 0.072 \\
\hline $\mathrm{PH}$ (echo. and/or RHC) & $5.01(3.18-7.89)$ & $<0.001$ & $4.15(2.59-6.65)$ & $<0.001$ \\
\hline $\mathrm{sPAP}<35 \mathrm{mmHg}$ & - & & - & \\
\hline $35-46 \mathrm{mmHg}$ & $2.05(0.98-4.28)$ & 0.056 & $1.26(0.58-2.70)$ & 0.559 \\
\hline$>46 \mathrm{mmHg}$ & $6.44(3.69-11.22)$ & $<0.001$ & $5.94(3.30-10.72)$ & $<0.001$ \\
\hline $\mathrm{PAH}(\mathrm{RHC})$ & $4.96(2.82-8.72)$ & $<0.001$ & $4.39(2.43-7.93)$ & $<0.001$ \\
\hline \multicolumn{5}{|l|}{ Heart involvement } \\
\hline Arrhythmia & $2.44(0.98-6.02)$ & 0.054 & $1.31(0.52-3.32)$ & 0.569 \\
\hline AV block & $0.95(0.13-6.80)$ & 0.956 & $1.15(0.15-8.58)$ & 0.890 \\
\hline BB block & $1.26(0.31-5.15)$ & 0.748 & $1.37(0.33-5.67)$ & 0.661 \\
\hline LVEF $<50 \%$ & $1.82(0.25-13.24)$ & 0.555 & $0.92(0.12-6.84)$ & 0.938 \\
\hline Diastolic dysfunction & $1.36(0.43-4.35)$ & 0.603 & $0.97(0.30-3.13)$ & 0.953 \\
\hline Pericarditis & $1.74(0.84-3.61)$ & 0.139 & $1.07(0.50-2.26)$ & 0.864 \\
\hline Valvular disease & $4.03(1.97-8.25)$ & $<0.001$ & $2.20(1.05-4.60)$ & 0.037 \\
\hline \multicolumn{5}{|l|}{ Renal involvement } \\
\hline Scleroderma renal crisis & $3.44(2.01-5.89)$ & $<0.001$ & $2.95(1.61-5.40)$ & $<0.001$ \\
\hline $\mathrm{GFR}<80 \mathrm{ml} / \mathrm{min}$ & $1.64(1.06-2.52)$ & 0.025 & $1.37(0.85-2.21)$ & 0.199 \\
\hline Gastrointestinal involvement & $1.07(0.68-1.69)$ & 0.756 & $1.02(0.65-1.62)$ & 0.916 \\
\hline $\mathrm{BMl}<18.5 \mathrm{~kg} / \mathrm{m} 2$ & $1.10(0.45-2.74)$ & 0.831 & $1.79(0.71-4.51)$ & 0.220 \\
\hline Albuminemia < $35 \mathrm{~g} / \mathrm{l}$ & $2.30(1.24-4.30)$ & 0.009 & $1.45(0.75-2.82)$ & 0.270 \\
\hline
\end{tabular}


Table 2 Prognosis factors: non-adjusted and adjusted analysis on age at diagnosis, sex, and SSc subtype in the French cohort (Continued)

\begin{tabular}{|c|c|c|c|c|}
\hline & Non-adjusted HR & $p$ & Adjusted HR & $p$ \\
\hline Muscular involvement & $1.66(1.10-2.51)$ & 0.016 & $1.46(0.92-2.31)$ & 0.106 \\
\hline CPK $>200 \mathrm{IU} / \mathrm{L}$ & $1.27(0.58-2.76)$ & 0.550 & $1.15(0.50-2.64)$ & 0.740 \\
\hline Joint involvement & $1.22(0.82-1.80)$ & 0.329 & $1.08(0.70-1.66)$ & 0.720 \\
\hline Cancer & $2.44(1.41-4.21)$ & 0.001 & $1.86(1.07-3.26)$ & 0.029 \\
\hline Anemia & $2.66(1.75-4.06)$ & $<0.001$ & $2.37(1.54-3.66)$ & $<0.001$ \\
\hline $\mathrm{CRP}>8 \mathrm{mg} / \mathrm{l}$ & $2.05(1.28-3.27)$ & 0.003 & $1.70(1.02-2.82)$ & 0.041 \\
\hline \multicolumn{5}{|l|}{ Serologic features } \\
\hline$A C A$ & $0.95(0.62-1.44)$ & 0.795 & $0.85(0.55-1.31)$ & 0.459 \\
\hline Anti-Scl70 antibodies & $0.87(0.55-1.36)$ & 0.534 & $0.82(0.51-1.30)$ & 0.390 \\
\hline Anti-U1RNP antibodies & $1.41(0.51-3.93)$ & 0.506 & $1.32(0.44-3.92)$ & 0.616 \\
\hline Anti-RNAP3 antibodies & $0.96(0.23-3.94)$ & 0.949 & $1.32(0.44-3.92)$ & 0.616 \\
\hline Anti-PMScl antibodies & $0.33(0.05-2.41)$ & 0.277 & $0.49(0.07-3.54)$ & 0.476 \\
\hline APL antibodies & $1.54(0.71-3.35)$ & 0.280 & $1.18(0.53-2.63)$ & 0.679 \\
\hline Low complement & $2.40(0.97-5.95)$ & 0.059 & $2.38(0.95-5.95)$ & 0.063 \\
\hline Smoking & $1.06(0.69-1.62)$ & 0.795 & $0.97(0.59-1.59)$ & 0.901 \\
\hline
\end{tabular}

Results are expressed as hazard ratios and $95 \%$ confidence interval

ICSSC limited cutaneous systemic sclerosis, dCSSC diffuse cutaneous systemic sclerosis, mRSS modified Rodnan score, GFR glomerular filtration rate, $A V$ block atrioventricular block, $B B$ block bundle branch block, $L V E F$ left ventricular ejection fraction, $P H$ pulmonary hypertension, $P A H$ pulmonary arterial hypertension, echo echocardiography, $R H C$ right heart catheterization, $6 M W D$ 6-min walking distance, sPAP systolic pulmonary arterial pressure, $H R C T$ high-resolution computer tomography, $T L C$ total lung capacity, FVC forced vital capacity, DLCO diffusing capacity of the lungs for carbon monoxide, $C R P C$ reactive protein, $B M I$ body mass index, $A C A$ anti-centromere antibodies, $A P L$ antiphospholipid antibodies

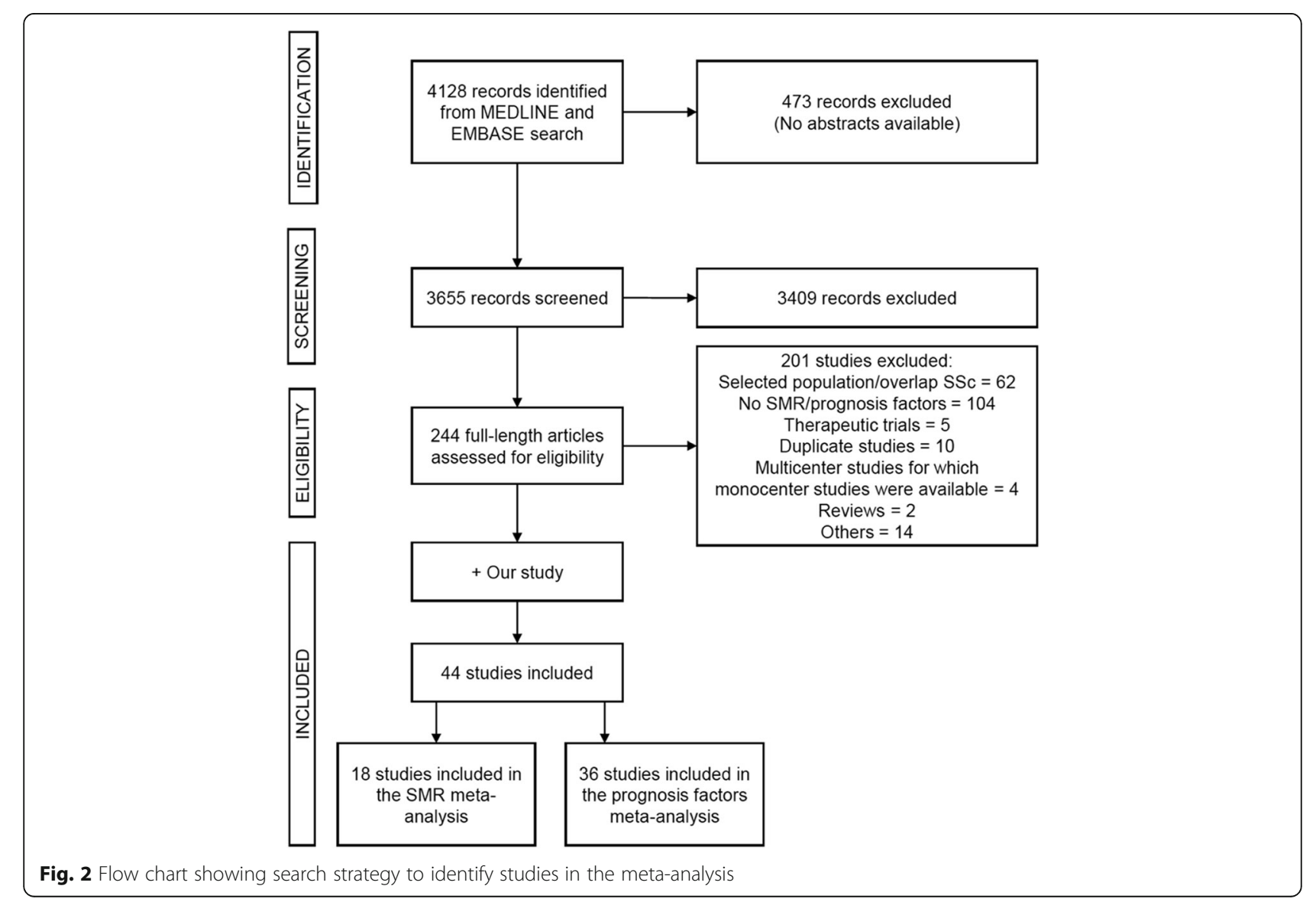


studies. Meta-regression analysis revealed a significant increase of SMR with proportion of dcSSc $(p<0.001)$ and prevalence of anti-Scl70 antibodies $(p=0.021)$. There was no association with male sex $(p=0.130)$. There was no significant association between SMR and mid-cohort year $(p=0.656)$ (Additional file 2: Figure S1. Prognosis factors meta-analysis).

\section{Prognosis factor meta-analysis}

Table 3 shows the results of the meta-analysis of prognosis factors. Age at disease onset, age at diagnosis, male sex, African origin, dcSSc, anti-Scl70 antibodies, renal involvement, scleroderma renal crisis, ILD, cardiac involvement, $\mathrm{PH}$, and cancer were significantly associated with a worse prognosis. The presence of $\mathrm{PH}$, diagnosed by Doppler echocardiography and/or RHC, was associated with a poor outcome (pooled HR $=3.44 ; 95 \%$ CI 2.59-4.58; $I^{2}=61.5 \%$; $p($ het $)=0.002)$. Meta-analysis of the five studies with $\mathrm{PH}$ defined by RHC revealed a pooled HR of 5.27 (95\% CI $2.98-9.31 ; I^{2}=63.7 \%$; $p$ (het) $=0.027$ ) for mortality. Heterogeneity could not be fully explained by the use of either echocardiography or $\mathrm{RHC}$ alone in defining $\mathrm{PH}$ as revealed by meta-regression stratified by the $\mathrm{PH}$ diagnosis method ( $p$ for residual heterogeneity $=0.012$ ). The presence of ACA was associated with a better survival, while the presence of joint involvement was not associated with prognosis (Additional file 2: Figure S2).

\section{Discussion}

The main results of our study are (i) a high risk of mortality in our cohort of incident patients, as shown by a high SMR of 5.73; (ii) the identification of age $>60$ years, dcSSc, dyspnea, $\mathrm{PH}$, low FVC, low DLCO, kidney involvement, valvular disease, cancer, telangiectasia, shorter $6 \mathrm{MWD}$, anemia, and inflammation as prognosis factors in our cohort; (iii) a high pooled SMR of 3.45 in the meta-analysis of the literature, including our new cohort; and (iv) the additional identification of male sex, African origin, ILD, cardiac involvement, and anti-Scl-70 antibodies as associated with worse prognosis in our meta-analysis, while ACA were associated with better prognosis.

\section{Survival and SMR}

With a mid-cohort year of 2008, our study population is the largest multicenter incident and well-phenotyped cohort study of SSc patients in France and is among the most recent published to date in the literature. The overall survival rates at 5 and 10 years from diagnosis were $85.9 \%$ and $71.7 \%$, respectively, and are lower than those reported in other recent cohorts [7, 22, 24, 44, 54]. We also report one of the highest SMR of 5.73. These differences could be explained by a high heterogeneity between studies as well as methodological issues such as the inclusion of prevalent cases in many studies or differences in time origin from which survival time is calculated (from disease onset, diagnosis, or enrolment). It is usually admitted that

Table 3 Results of the meta-analysis of prognosis factors in SSC

\begin{tabular}{|c|c|c|c|c|c|c|}
\hline & Number of cohorts & $\mathrm{HR}$ & $95 \% \mathrm{Cl}$ & $R^{2}(\%)$ & $p$ (het) & $\overline{\text { Egger's test }}$ \\
\hline Age at disease onset (per 1 year) & 6 & 1.05 & $(1.04-1.07)$ & 68.6 & 0.007 & 0.783 \\
\hline Age at diagnosis (per 1 year) & 5 & 1.04 & $(1.04-1.05)$ & 71.2 & 0.008 & 0.025 \\
\hline Male sex & 21 & 1.87 & $(1.61-2.18)$ & 50.9 & 0.004 & $<0.001$ \\
\hline African origin & 5 & 1.38 & $(1.15-1.66)$ & 25.0 & 0.255 & 0.774 \\
\hline dcSSc & 23 & 1.90 & $(1.62-2.23)$ & 58.3 & $<0.001$ & $<0.001$ \\
\hline Anti-Scl70 autoantibodies & 13 & 1.38 & $(1.09-1.74)$ & 49.6 & 0.022 & 0.024 \\
\hline ACA & 8 & 0.62 & $(0.47-0.82)$ & 56.4 & 0.025 & 0.590 \\
\hline Joint involvement & 4 & 1.32 & $(0.82-2.12)$ & 54.0 & 0.089 & 0.508 \\
\hline Renal involvement & 9 & 2.79 & $(1.95-3.99)$ & 50.9 & 0.039 & 0.512 \\
\hline Scleroderma renal crisis & 10 & 3.89 & $(2.38-6.36)$ & 75.6 & $<0.001$ & 0.097 \\
\hline ILD & 14 & 2.34 & $(1.78-3.08)$ & 69.5 & $<0.001$ & $<0.001$ \\
\hline Cardiac involvement & 7 & 4.35 & $(2.28-8.29)$ & 89.9 & $<0.001$ & 0.077 \\
\hline $\begin{array}{l}\text { PH (echocardiography } \\
\text { or RHC) }\end{array}$ & 13 & 3.44 & $(2.59-4.58)$ & 61.5 & 0.002 & 0.057 \\
\hline $\mathrm{PH}(\mathrm{RHC})$ & 5 & 5.27 & $(2.98-9.31)$ & 63.7 & 0.027 & 0.761 \\
\hline Cancer & 6 & 2.11 & $(1.27-3.50)$ & 76.2 & $<0.001$ & 0.016 \\
\hline
\end{tabular}

Results are expressed as hazard ratios with $95 \%$ confidence interval. The $l^{2}$ statistics describes the percentage of variation across studies that is due to heterogeneity rather than chance. $p$ (het) is the $p$ value for the ${ }^{2}$ test for heterogeneity. Egger's test checks for funnel plot asymmetry $d c S S c$ diffuse cutaneous systemic sclerosis, ILD interstitial lung disease, $A C A$ anti-centromere antibodies, $P H$ pulmonary hypertension, $R H C$ right heart catheterization 
studies including prevalent cases underestimate mortality and that better survival is observed in prevalent patients with longer disease duration prior to inclusion. Yet, our meta-analysis did not show a significant difference between pooled SMR of studies that included prevalent cases and those restricted to incident according to the authors' definition. The high heterogeneity observed within studies with incident patients could be due to the definition of incidence and the proportion of males and patients with anti-Scl70 antibodies. This high heterogeneity could explain the lack of difference between incident and prevalent cohorts. Meta-regression showed a significant association between SMR and proportion of dcSSc $(p<0.001)$ and prevalence of anti-Scl70 antibodies $(p=0.021)$. Our high SMR of 5.73 could therefore be partly explained by the high proportion of anti-Scl70 antibodies $(35 \%)$ in our population. Interestingly, there has been a debate whether or not the survival could have improved over time in SSc. Our study did not show any improvement of SMR over time, which is in line with the study of Elhai et al. [6]. However, considering life expectancy in OECD (Organisation for Economic Co-operation and Development) countries increased by 12 years from 1960 to 2014 [56], and SMR being the ratio of mortality in SSc cohorts to that of the general population, this suggests that all-cause mortality has decreased proportionately to the general population in SSc cohorts.

\section{Prognosis factors}

Prognosis factors have been assessed in many observational studies [7, 13-26] and have been recently reviewed. Our systematic review and meta-analysis, as well as two prior meta-analyses [5, 27] and a recent EUSTAR study [2], have identified the following characteristics as consistently associated with a worse prognosis: male gender; older age; dcSSc; lung and cardiac involvement, including $\mathrm{PH}$ and ILD; kidney involvement; and inflammation. These robust factors are included in a recent prognosis score [2] as well as in older ones [57, 58].

Besides these well-known prognosis factors, our cohort study identifies new ones: telangiectasia, 6MWD, valvular disease, cancer, and autoantibody status.

Telangiectasia was slightly associated with a higher mortality in our study population. In contrast, Poormoghim et al. [51] reported a non-significant, yet elevated HR of 1.44 in a smaller cohort of Iranian patients. An increased number of telangiectasia has been suggested to be a clinical marker of microvascular disease in SSc and is associated with an increased risk of PAH [59].

The 6MWD is a simple tool used to assess submaximal functional capacity. It is influenced by various disease parameters during SSc and lacks organ specificity [60]. While the 6MWD has been shown to be an independent predictor of mortality in idiopathic PAH, its prognosis value in SSc-PAH is less clear [61]. To our knowledge, we are the first to report a negative association of the 6MWD with survival $(\mathrm{HR}=0.51)$ in SSc patients. This can be at least partly explained by the association between the 6MWD and PAH [60].

While cardiac involvement in SSc patients is robustly associated with a poor prognosis, conferring a nearly fivefold increased risk of mortality in our meta-analysis, no study had yet focused on the valvular manifestations of SSc. A recent article comparing echocardiography in SSc patients and a matched control population showed a greater frequency of valvular regurgitation and valvular replacement due to regurgitation [62]. Moreover, in a large multicenter French cohort, De Groote et al. [63] reported $6.7 \%$ mitral regurgitation and $2.5 \%$ aortic regurgitations. To our knowledge, we are the first to describe an association between valvular disease and survival in SSc. These data indicate that more attention should be paid to valvular disease in SSc patients and further studies are needed to confirm its prognostic significance.

An increased incidence of malignancy has been reported during SSc, especially lung and hematological cancer [64]. Cancer has also been described among the leading cause of non-SSc-related deaths [7, 11, 15, 65], and a temporal relation has been reported between the onset of cancer and SSc [66]. As expected, in our cohort as well as in the meta-analysis, malignancy was significantly associated with shorter survival.

In our cohort, we did not observe any association between anti-Scl70 antibodies or ACA and survival. Interestingly, the absence of association of anti-Scl70 antibodies with survival has been suggested by numerous studies $[7,17,18,23,25]$ while the protective role of anti-centromere antibodies is better established [7, 18, $22,25,48]$. Our meta-analysis confirms that the presence of ACA is associated with better survival (pooled $\mathrm{HR}=0.58$ ). Moreover, our meta-analysis suggests that the presence of anti-Scl70 antibodies could be indeed a predictor of mortality with a pooled HR of 1.38. Our analysis also highlights a probable publication bias. Small studies reporting a negative association of anti-Scl70 antibodies with death are notably lacking. Therefore, it is difficult to draw a firm conclusion on the role of anti-Scl70 antibodies as a prognosis factor in SSc.

The major strength of our study is the availability of detailed clinical and laboratory characteristics in a multicenter cohort of incident patients. The major strengths of our meta-analysis include (i) the first analysis of pooled HR of anti-Scl70/ACA antibodies, (ii) the separate analysis of pooled SMR in incident cohorts of SSc, and (iii) the separate analysis of pooled HR of PH diagnosed by RHC only.

The main limitation is a proportion of loss to follow-up of around $20 \%$ in our cohort, despite our 
attempts to collect information on participants who dropped out. These patients lost to follow-up had a higher prevalence of ILD and lower prevalence of ACA at baseline, leading to potential underestimation of mortality. A second limitation is the variable definition of incident patients among studies. Although we defined incident patients as newly diagnosed ones, these patients had relatively short disease duration from first non-RP symptom diagnosis, thus minimizing survivor bias. Because of missing values, the effect of specific treatments and other data such as type and stage of cancer could not be studied, and no multivariate analysis could be performed. In addition, multiple univariate tests are responsible for an inflation of the alpha risk. However, multiple test adjustments in such exploratory study, in addition for a rare disease, are not strictly required [67]. Finally, our study was performed in five selected referral centers and may therefore have focused on a subset of patients with more severe disease, which could limit the representativeness of our findings.

\section{Conclusions}

Our results show that mortality is still high in SSc. Strong prognosis factors identified at baseline are age at diagnosis $>60$ years, dcSSc subtype, scleroderma renal crisis, severe dyspnea, FVC and DLCO $<70 \%, \mathrm{PH}$, anemia, and CRP $>8 \mathrm{mg} / \mathrm{l}$. Our study also suggests the prognosis value of telangiectasia, 6MWD, valvular disease, cancer, and autoantibody status.

\section{Additional files}

Additional file 1: Table S1. Survival rates and survival curve for ICSSC and dcSSc. Table S2. Main characteristics of studies in the SMR metaanalysis. Table S3. Main characteristics of studies in the prognosis factors meta-analysis. (DOCX 51 kb)

Additional file 2: Figure S1. Funnel plots, forest plots, and metaregression for the meta-analysis of SMR. Figure S2. Funnel plots and forest plots of the risk factors related with mortality. (PDF $948 \mathrm{~kb}$ )

\section{Abbreviations}

6MWD: 6-min walking distance; ACA: Anti-centromere antibodies; APL: Antiphospholipid antibodies; AV block: Atrioventricular block; BB block: Bundle branch block; BMI: Body mass index; CPK: Creatinine kinase; CRP: C reactive protein; dcSSc: Diffuse cutaneous systemic sclerosis; DLCO: Diffusing capacity of the lungs for carbon monoxide; FVC: Forced vital capacity; ILD: Interstitial lung disease; ICSSc: Limited cutaneous systemic sclerosis; LVEF: Left ventricular ejection fraction; mRSS: Modified Rodnan skin score; OECD: Organisation for Economic Co-operation and Development; PAH: Pulmonary arterial hypertension; PAP: Pulmonary arterial pressure; PH: Pulmonary hypertension; RHC: Right heart catheterization; RP: Raynaud phenomenon; SMR: Standardized mortality ratio; SSc: Systemic sclerosis; TLC: Total lung capacity

\section{Acknowledgements}

French National Scleroderma Cohort coauthors: Zahir AMOURA Z, Olivier AUMAITRE, Eric AUXENFANTS, Marie-Hélène BALQUET, Cristina BELIZNA, Alice BEREZNE A, Bernard BONNOTTE, Pascal CATHEBRAS, Emmanuel CHATELUS, Joël CONSTANS, Vincent COTTIN V, Gonzalo DE LUNA G, Robin DHOTE, Elisabeth DIOT, Olivier FAIN, Anne-Laure FAUCHAIS, Yves FRANCES,
Jean-Gabriel FUZIBET, Jean-Baptiste GAULTIER, Tiphaine GOULENOK, Brigitte GRANEL, Jean-Robert HARLE, Pierre-Yves HATRON, Arnaud HOT, Bernard IMBERT,

Jean-Emmanuel KAHN, Gilles KAPLANSKI, Pierre KIEFFER, Noémie LE GOUELLEC, Alain LE QUELLEC, Olivier LIDOVE, Nadine MAGY-BERTRAND, François MAURIER, Sylvain PALAT, Thomas PAPO, Jean-Louis

PENNAFORTE, Jacques POUCHOT, Grégory PUGNET, Thomas QUEMENEUR, Viviane QUEYREL, Laurent SAILLER, Thierry SCHAEVERBEKE, Marie-Elise TRUCHETET, Denis WAHL.

\section{Funding}

Grants or financial supporters: none.

Financial support or other benefits from commercial sources: none.

\section{Availability of data and materials}

The datasets generated and/or analysed during the current study are not publicly available due data protection regulations but are available from the corresponding author on reasonable request.

\section{Authors' contributions}

MRP performed the systematic review of the literature and was a major contributor in interpreting the cohort data and meta-analysis and in writing the manuscript. JG was a major contributor in the design of the cohort study, performed data management, analyzed the cohort data, and was a major contributor in the interpretation of data. LD performed the statistical tests of the meta-analysis and was a major contributor in the interpretation of data. $L M, C A, J C L, Y A, B B, S B$, and $A M$ were significant contributors in the acquisition of data and in the critical review of the manuscript. EH was a major contributor in the design of the study, in the interpretation of data, and in the critical review of the manuscript. DL was a major contributor in the design of the study, in the systematic review of the literature, in the interpretation of data, and in writing the manuscript. All authors read and approved the final manuscript.

\section{Ethics approval and consent to participate}

This study was authorized by the French Ministry of Research. The authorization number is 13145. To issue such authorization, the Ministry of Research has sought the advice of an independent ethics committee, namely the "Comité consultatif sur le traitement de l'information en matière de recherche (CCTIRS)," which voted positively. French legislation on non-interventional studies requires collecting the non-opposition of patients but does not require written consent. As such, non-opposition was obtained from each patient included in the study for the use of their de-identified medical record data.

\section{Consent for publication}

Not applicable.

\section{Competing interests}

The authors declare that they have no competing interests.

\section{Publisher's Note}

Springer Nature remains neutral with regard to jurisdictional claims in published maps and institutional affiliations.

\section{Author details}

'University of Lille, U995 - LIRIC - Lille Inflammation Research International Center, F-59000 Lille, France. ${ }^{2}$ INSERM, U995, F-59000 Lille, France. ${ }^{3} \mathrm{CHU}$ Lille, Département de Médecine Interne et Immunologie Clinique, F-59000 Lille, France. ${ }^{4}$ Centre de Référence des Maladies Autoimmunes et Systémiques Rares du Nord et Nord-Ouest de France (CeRAINO), Lille, France. ${ }^{5}$ Inserm UMR1 167, RID-AGE, Risk Factors and Molecular Determinants of Aging-Related Diseases, Université de Lille, Centre Hosp. Univ Lille, Institut Pasteur de Lille, Lille, France. 'Service de Médecine Interne, Hôpital Cochin, Centre de Référence pour les Maladies Systémiques Autoimmunes Rares d'lle de France, Université Paris Descartes, Sorbonne Paris Cité, Assistance Publique-Hôpitaux de Paris (AP-HP), Paris, France. ${ }^{7} \mathrm{CHU}$ Nantes, Service de Médecine Interne, Nantes, France. ${ }^{8}$ Department of Internal and Vascular Medicine, Centre Hospitalier Lyon Sud, Hospices Civils de Lyon, Pierre-Bénite, France. ${ }^{9}$ Univ Lyon, UMR 5558, Laboratoire de Biométrie et Biologie Evolutive, CNRS, Claude Bernard University, F-69003 Lyon, France. ${ }^{10}$ Hôpital 
Cochin-APHP-Service de Rhumatologie A, Université Paris Descartes, INSERM U1016, Paris, France. ${ }^{11}$ INSERM U 1085 (IRSET), University of Rennes 1, Rennes, France. ${ }^{12}$ Service de Médecine Interne CHU Caen, Caen, France. ${ }^{13}$ Service de Médecine Interne et Immunologie Clinique, CHU Dijon, Dijon, France.

${ }^{14}$ Hôpital Saint-Antoine-APHP-Service de Médecine Interne, Paris, France.

Received: 19 November 2018 Accepted: 18 March 2019

Published online: 03 April 2019

\section{References}

1. Steen VD, Medsger TA. Changes in causes of death in systemic sclerosis, 19722002. Ann Rheum Dis. 2007;66:940-4.

2. Elhai M, Meune C, Boubaya M, Avouac J, Hachulla E, Balbir-Gurman A, et AL Mapping and predicting mortality from systemic sclerosis. Ann Rheum Dis. 2017:76:1897-1905.

3. Al-Dhaher FF, Pope JE, Ouimet JM. Determinants of morbidity and mortality of systemic sclerosis in Canada. Semin Arthritis Rheum. 2010;39:269-77.

4. Nihtyanova SI, Tang EC, Coghlan JG, Wells AU, Black CM, Denton CP. Improved survival in systemic sclerosis is associated with better ascertainment of internal organ disease: a retrospective cohort study. QJM. 2010;103:109-15.

5. Rubio-Rivas M, Royo C, Simeón CP, Corbella X, Fonollosa V. Mortality and survival in systemic sclerosis: systematic review and meta-analysis. Semin Arthritis Rheum. 2014:44:208-19.

6. Elhai M, Meune C, Avouac J, Kahan A, Allanore Y. Trends in mortality in patients with systemic sclerosis over 40 years: a systematic review and metaanalysis of cohort studies. Rheumatology. 2012;51:1017-26.

7. Hao Y, Hudson M, Baron M, Carreira P, Stevens W, Rabusa C, et al. Early mortality in a multinational systemic sclerosis inception cohort. Arthritis Rheumatol. 2017;69:1067-77.

8. Alamanos Y, Tsifetaki N, Voulgari P, Siozos C, Tsamandouraki K, Alexiou G, et al. Epidemiology of systemic sclerosis in northwest Greece 1981 to 2002. Semin Arthritis Rheum. 2005:34:714-20.

9. Bryan C, Howard Y, Brennan P, Black CM, Silman A. Survival following the onset of scleroderma: results from a retrospective inception cohort study of the UK patient population. Rheumatology. 1996;35:1122-6.

10. Loannidis JPA, Vlachoyiannopoulos PG, Haidich A-B, Medsger TA, Lucas M, Michet $C J$, et al. Mortality in systemic sclerosis: an international metaanalysis of individual patient data. Am J Med. 2005;118:2-10.

11. Jacobsen S, Halberg P, Ullman S. Mortality and causes of death of 344 Danish patients with systemic sclerosis (scleroderma). Rheumatology. 1998:37:750-5.

12. Kuo C-F, See L-C, Yu K-H, Chou I-J, Tseng W-Y, Chang H-C, et al. Epidemiology and mortality of systemic sclerosis: a nationwide population study in Taiwan. Scand J Rheumatol. 2011:40:373-8.

13. Lee $P$, Langevitz $P$, Alderdice $C$, Aubrey M, Baer P, Baron M, et al. Mortality in systemic sclerosis (scleroderma). QJM. 1992;82:139-48.

14. Ferri C, Valentini G, Cozzi F, Sebastiani M, Michelassi C, La Montagna G, et al. Systemic sclerosis: demographic, clinical, and serologic features and survival in 1,012 Italian patients. Medicine (Baltimore). 2002;81:139-53.

15. Scussel-Lonzetti L, Joyal F, Raynauld J-P, Roussin A, Rich E, Goulet J-R, et al. Predicting mortality in systemic sclerosis: analysis of a cohort of 309 French Canadian patients with emphasis on features at diagnosis as predictive factors for survival. Medicine (Baltimore). 2002;81:154-67.

16. Czirjak L, Kumanovics G, Varju C, Nagy Z, Pakozdi A, Szekanecz Z, et al, Survival and causes of death in 366 Hungarian patients with systemic sclerosis. Ann Rheum Dis. 2008;67:59-63.

17. Hachulla E, Carpentier P, Gressin V, Diot E, Allanore Y, Sibilia J, et al. Risk factors for death and the 3-year survival of patients with systemic sclerosis: the French ItinerAIR-Sclerodermie study. Rheumatology. 2008;48:304-8.

18. ssassi S, del Junco D, Sutter K, McNearney TA, Reveille JD, Karnavas A, et al. Clinical and genetic factors predictive of mortality in early systemic sclerosis. Arthritis Rheum. 2009;61:1403-11.

19. Joven BE, Almodovar R, Carmona L, Carreira PE. Survival, causes of death, and risk factors associated with mortality in Spanish systemic sclerosis patients: results from a single university hospital. Semin Arthritis Rheum. 2010;39:285-93.

20. Kim J, Park SK, Moon KW, Lee EY, Lee YJ, Song YW, et al. The prognostic factors of systemic sclerosis for survival among Koreans. Clin Rheumatol. 2010;29:297-302

21. Sampaio-Barros PD, Bortoluzzo AB, Marangoni RG, Rocha LF, Del Rio APT, Samara AM, et al. Survival, causes of death, and prognostic factors in systemic sclerosis: analysis of 947 Brazilian patients. J Rheumatol. 2012:39:1971-8

22. Hoffmann-Vold A-M, Molberg $\varnothing$, Midtvedt $\varnothing$, Garen T, Gran JT. Survival and causes of death in an unselected and complete cohort of Norwegian patients with systemic sclerosis. J Rheumatol. 2013;40:1127-33.

23. Strickland G, Pauling J, Cavill C, Shaddick G, McHugh N. Mortality in systemic sclerosis - a single centre study from the UK. Clin Rheumatol. 2013; 32:1533-9.

24. Alba MA, Velasco C, Simeón CP, Fonollosa V, Trapiella L, Egurbide MV et al. Early- versus late-onset systemic sclerosis: differences in clinical presentation and outcome in 1037 patients. Medicine (Baltimore). 2014; 93:73-81.

25. Cottrell TR, Wise RA, Wigley FM, Boin F. The degree of skin involvement identifies distinct lung disease outcomes and survival in systemic sclerosis. Ann Rheum Dis. 2014:73:1060-6.

26. Nihtyanova SI, Schreiber BE, Ong VH, Rosenberg D, Moinzadeh $\mathrm{P}$, Coghlan JG, et al. Prediction of pulmonary complications and long-term survival in systemic sclerosis: pulmonary complications and survival in SSc. Arthritis Rheumatol. 2014:66:1625-35.

27. Komócsi A, Vorobcsuk A, Faludi R, Pintér T, Lenkey Z, Költő G, et al. The impact of cardiopulmonary manifestations on the mortality of SSC: a systematic review and meta-analysis of observational studies. Rheumatology. 2012:51:1027-36.

28. Preliminary criteria for the classification of systemic sclerosis (scleroderma). Subcommittee for scleroderma criteria of the American Rheumatism Association Diagnostic and Therapeutic Criteria Committee. Arthritis Rheum. 1980;23:581-90.

29. van den Hoogen F, Khanna D, Fransen J, Johnson SR, Baron M, Tyndall A, et al. 2013 classification criteria for systemic sclerosis: an American College of Rheumatology/European League against rheumatism collaborative initiative: ACR/EULAR classification criteria for SSc. Arthritis Rheum. 2013;65:2737-47.

30. Nagueh SF, Smiseth OA, Appleton CP, Byrd BF, Dokainish H, Edvardsen $T$, et al. Recommendations for the evaluation of left ventricular diastolic function by echocardiography: an update from the American Society of Echocardiography and the European Association of Cardiovascular Imaging. J Am Soc Echocardiogr. 2016;29:277-314.

31. Stroup DF, Berlin JA, Morton SC, et al. Meta-analysis of observational studies in epidemiology: a proposal for reporting. JAMA. 2000;283:2008-12.

32. Wells $G$, Shea B, O'Connell D, Peterson J, Welch V, Losos M, et al. The Newcastle-Ottawa scale (NOS) for assessing the quality of nonrandomized studies in meta-analysis [internet]. Available from: http://www.ohri.ca/ programs/clinical_epidemiology/oxford.asp.

33. Abu-Shakra M, Lee P. Mortality in systemic sclerosis: a comparison with the general population. J Rheumatol. 1995;22:2100-2.

34. Cruz-Domínguez MP, García-Collinot G, Saavedra MA, Montes-Cortes DH, Morales-Aguilar R, Carranza-Muleiro RA, et al. Malnutrition is an independent risk factor for mortality in Mexican patients with systemic sclerosis: a cohort study. Rheumatol Int. 2017:37:1101-9.

35. Hesselstrand R, Scheja A, Akesson A. Mortality and causes of death in a Swedish series of systemic sclerosis patients. Ann Rheum Dis. 1998;57:682-6.

36. Mok CC, Kwok CL, Ho LY, Chan PT, Yip SF. Life expectancy, standardized mortality ratios, and causes of death in six rheumatic diseases in Hong Kong, China. Arthritis Rheum. 2011;63:1182-9.

37. Perez-Bocanegra C, Solans-Laque R, Simeon-Aznar CP, Campillo M, Fonollosa-Pla V, Vilardell-Tarres M. Age-related survival and clinical features in systemic sclerosis patients older or younger than 65 at diagnosis. Rheumatology. 2010;49:1112-7.

38. Zarafonetis CJ, Dabich L, Negri D, Skovronski JJ, Devol EB, Wolfe R. Retrospective studies in scleroderma: effect of potassium paraaminobenzoate on survival. J Clin Epidemiol. 1988:41:193-205.

39. Beretta L, Santaniello A, Cappiello F, Chawla NV, Vonk MC, Carreira PE, et al. Development of a five-year mortality model in systemic sclerosis patients by different analytical approaches. Clin Exp Rheumatol. 2010;28:S18.

40. Beretta L, Cappiello F, Barili M, Scorza R. Proximal interleukin-10 gene polymorphisms in Italian patients with systemic sclerosis. Tissue Antigens. 2007;69:305-12

41. Bernal-Bello D, de Tena JG, Guillén-del Castillo A, Selva-O'Callaghan A, Callejas-Moraga EL, Marín-Sánchez AM, et al. Novel risk factors related to cancer in scleroderma. Autoimmun Rev. 2017:16:461-8. 
42. Codullo V, Cereda E, Klersy C, Cavazzana I, Alpini C, Bonardi C, et al. Serum prealbumin is an independent predictor of mortality in systemic sclerosis outpatients. Rheumatology. 2016;55:315-9.

43. Fernández-Codina A, Simeón-Aznar CP, Pinal-Fernandez I, RodríguezPalomares J, Pizzi MN, Hidalgo CE, et al. Cardiac involvement in systemic sclerosis: differences between clinical subsets and influence on survival. Rheumatol Int. 2017;37:75-84.

44. Ferri C, Sebastiani M, Lo Monaco A, ludici M, Giuggioli D, Furini F, et al. Systemic sclerosis evolution of disease pathomorphosis and survival. Our experience on Italian patients' population and review of the literature. Autoimmun Rev. 2014;13:1026-34.

45. Gelber AC, Manno RL, Shah AA, Woods A, Le EN, Boin F, et al. Race and association with disease manifestations and mortality in scleroderma. Medicine (Baltimore). 2013;92:191-205.

46. Hesselstrand R. The association of antinuclear antibodies with organ involvement and survival in systemic sclerosis. Rheumatology. 2003;42: 534-40.

47. Hinchcliff M, Desai CS, Varga J, Shah SJ. Prevalence, progn,osis, and factors associated with left ventricular diastolic dysfunction in systemic sclerosis. Clin Exp Rheumatol. 2012;30:S30.

48. Hussein $H$, Lee $P$, Chau $C$, Johnson SR. The effect of male sex on survival in systemic sclerosis. J Rheumatol. 2014;41:2193-200.

49. Költő G, Faludi R, Aradi D, Bartos B, Kumánovics G, Minier T, et al. Impact of cardiac involvement on the risk of mortality among patients with systemic sclerosis: a 5-year follow-up of a single-center cohort. Clin Rheumatol. 2014; 33:197-205.

50. Mayes MD, Lacey JV, Beebe-Dimmer J, Gillespie BW, Cooper B, Laing TJ, et al. Prevalence, incidence, survival, and disease characteristics of systemic sclerosis in a large US population. Arthritis Rheum. 2003;48:2246-55.

51. Poormoghim H, Andalib E, Jalali A, Ghaderi A, Ghorbannia A, Mojtabavi N. Survival and causes of death in systemic sclerosis patients: a single center registry report from Iran. Rheumatol Int. 2016;36:925-34.

52. Ruangjutipopan S, Kasitanon N, Louthrenoo W, Sukitawut W, Wichainun R. Causes of death and poor survival prognostic factors in thai patients with systemic sclerosis. J Med Assoc Thail Chotmaihet Thangphaet. 2002;85: 1204-9.

53. Simeon CP. Mortality and prognostic factors in Spanish patients with systemic sclerosis. Rheumatology. 2003;42:71-5.

54. Simeón-Aznar CP, Fonollosa-Plá V, Tolosa-Vilella C, Espinosa-Garriga G, Campillo-Grau M, Ramos-Casals M, et al. Registry of the Spanish network for systemic sclerosis: survival, prognostic factors, and causes of death. Medicine (Baltimore). 2015;94:e1728.

55. Steen V, Domsic RT, Lucas M, Fertig N, Medsger TA. A clinical and serologic comparison of African American and Caucasian patients with systemic sclerosis. Arthritis Rheum. 2012;64:2986-94.

56. OECD. "Life expectancy at birth", in Health at a Glance 2017: OECD Indicators [Internet]. OECD Publishing (Paris); Available from: https://doi.org/ 10.1787/health_glance-2017-6-en

57. Fransen J, Popa-Diaconu D, Hesselstrand R, Carreira P, Valentini G, Beretta L, et al. Clinical prediction of 5-year survival in systemic sclerosis: validation of a simple prognostic model in EUSTAR centres. Ann Rheum Dis. 2011;70: 1788-92.

58. Bryan C, Knight C, Black CM, Silman AJ. Prediction of five-year survival following presentation with scleroderma: development of a simple model using three disease factors at first visit. Arthritis Rheum. 1999:42:2660-5.

59. Jouvray M, Launay D, Dubucquoi S, Sobanski V, Podevin C, Lambert M, et al. Whole-body distribution and clinical association of Telangiectases in systemic sclerosis. JAMA Dermatol. 2018;154:796-805.

60. Sanges S, Giovannelli J, Sobanski V, Morell-Dubois S, Maillard H, Lambert M, et al. Factors associated with the 6-minute walk distance in patients with systemic sclerosis. Arthritis Res Ther. 2017;19:279-90.

61. Lefèvre G, Dauchet L, Hachulla E, Montani D, Sobanski V, Lambert M, et al. Survival and prognostic factors in systemic sclerosis-associated pulmonary hypertension: a systematic review and meta-analysis. Arthritis Rheum. 2013; 65:2412-23.

62. Nordin A, Svenungsson E, Björnådal L, Elvin K, Larsson A, Jensen-Urstad K. Troponin I and echocardiography in patients with systemic sclerosis and matched population controls. Scand J Rheumatol. 2017:46:226-35.

63. de GP, Gressin V, Hachulla E, Carpentier P, Guillevin L, Kahan A, et al. Evaluation of cardiac abnormalities by Doppler echocardiography in a large nationwide multicentric cohort of patients with systemic sclerosis. Ann Rheum Dis. 2008;67:31-6.

64. Onishi A, Sugiyama D, Kumagai S, Morinobu A. Cancer incidence in systemic sclerosis: meta-analysis of population-based cohort studies. Arthritis Rheum. 2013;65:1913-21.

65. Tyndall AJ, Bannert B, Vonk M, Airo P, Cozzi F, Carreira PE, et al. Causes and risk factors for death in systemic sclerosis: a study from the EULAR Scleroderma Trials and Research (EUSTAR) database. Ann Rheum Dis. 2010; 69:1809-15.

66. Shah AA, Rosen A, Hummers L, Wigley F, Casciola-Rosen L. Close temporal relationship between onset of cancer and scleroderma in patients with RNA polymerase I/III antibodies. Arthritis Rheum. 2010;62:2787-95.

67. Bender R, Lange S. Adjusting for multiple testing - when and how? J Clin Epidemiol. 2001;54:343-9.

\section{Ready to submit your research? Choose BMC and benefit from:}

- fast, convenient online submission

- thorough peer review by experienced researchers in your field

- rapid publication on acceptance

- support for research data, including large and complex data types

- gold Open Access which fosters wider collaboration and increased citations

- maximum visibility for your research: over $100 \mathrm{M}$ website views per year

At BMC, research is always in progress.

Learn more biomedcentral.com/submissions 